\title{
Detection value of tumor cells in cerebrospinal fluid in the diagnosis of meningeal metastasis from lung cancer by immuno-FISH technology
}

\author{
YUAN LV*, NING MU*, CHUNHUA MA, RONG JIANG, QIAOLI WU, JINDUO LI, BIN WANG and LIWEI SUN \\ Department of Intervention, Tianjin Key Laboratory of Cerebral Vascular and Neurodegenerative Diseases, \\ Tianjin Huanhu Hospital, Tianjin 300350, P.R. China
}

Received May 31, 2015; Accepted September 29, 2016

DOI: $10.3892 / \mathrm{ol} .2016 .5314$

\begin{abstract}
To investigate the detection value of tumor cells in cerebrospinal fluid (CSF) in the adjuvant diagnosis of meningeal metastasis from lung cancer by immunofluorescence in situ hybridization (immuno-FISH) technology. The circulating tumor cells (CTCs) in the CSF of 16 patients with meningeal metastasis from lung cancer and 8 with non-tumor diseases in the brain were detected using immuno-FISH technology. The diagnosis of meningeal metastasis from lung cancer was based on neurological symptoms, enhanced magnetic resonance imaging (MRI) scans and CSF cytological examination. The number of CTCs in the patients with meningeal metastasis from lung cancer was significantly higher than those with non-tumor diseases in the brain $(\mathrm{P}<0.01)$. The critical point of the maximum correct diagnostic index (Youden index) was regarded as the judging criterion for positive tumor cells in CSF according to the receiver operating characteristic curve. When there was one tumor cell in $7.5 \mathrm{ml}$ CSF, the area under curve was 0.875 (95\% confidence interval, $0.705 \sim 1.000)$. The diagnostic sensitivity, specificity, effectiveness, positive and negative predictive values were 75.0, 100.0, $83.3,100.0$ and $66.7 \%$, respectively. There may be great clinical value in the detection of CTCs in CSF for the diagnosis of meningeal metastasis from lung cancer by immuno-FISH technology.
\end{abstract}

Correspondence to: Dr Rong Jiang, Department of Intervention, Tianjin Key Laboratory of Cerebral Vascular and Neurodegenerative Diseases, Tianjin Huanhu Hospital, 6 Jizhao Road, Tianjin 300350, P.R. China

E-mail: jiangrong1999@126.com

*Contributed equally

Key words: circulating tumor cells, lung cancer, meningeal metastasis, diagnosis, immunofluorescence in situ hybridization

\section{Introduction}

Meningeal metastasis is a metastatic carcinoma of the central nervous system caused by diffuse dissemination or focal infiltration of malignant tumor cells in meninges and spinal subarachnoid space, secondary to leukemia, lymphoma, lung cancer and breast cancer (1). The disease is characterized by rapid progression and poor prognosis (2). The median survival time of patients with meningeal metastasis, who did not undergo treatment, was only 4-6 weeks (2). Therefore, to seek a sensitive method in the detection of potential meningeal metastasis is extremely necessary. Previous studies have identified that blood circulating tumor cells (CTCs), namely solid tumor cells falling into the blood, have a high correlation with tumor diagnosis (3), metastasis and prognosis $(4,5)$. In the present study, CTCs in the cerebrospinal fluid (CSF) of patients with meningeal metastasis from lung cancer were detected using immunofluorescence in situ hybridization (immuno-FISH) technology and the detection value of the CTCs were evaluated in the adjuvant diagnosis of meningeal metastasis from lung cancer. The judging criterion for CTCs in the optimal CSF was selected to improve the early diagnostic rate of meningeal metastasis from lung cancer.

\section{Materials and methods}

General patient data. Patients in the present study were enrolled at Tianjin Huanhu Hospital (Tianjin, China). The inclusion criteria was as follows: i) Patients with non-small cell lung cancer definitely diagnosed by histology or cytology; ii) patients diagnosed with meningeal metastasis*; iii) no intracranial metastatic lesions with a diameter $>1 \mathrm{~cm}$ determined via magnetic resonance imaging (MRI); iv) no history of encephalitis and craniocerebral trauma within 6 months, and no brain surgery or radiotherapy; v) controllable intracranial hypertension following treatment with dehydrated drugs; vi) tolerable to lumber puncture in the collection of CSF; vii) exclusion of patients complicated by cerebrospinal lesions, including intracranial meningioma, ependymoma and spinal meningioma; and viii) providing informed consent. According to the aforementioned inclusion criteria, a total of 16 patients were enrolled, in which there were 2 males and 14 females 
Table I. Detection results of circulating tumor cells based on different judging criteria $(\bar{x} \pm s)$ (number of tumor cells/7.5 ml cerebrospinal fluid).

\begin{tabular}{lcccc}
\hline Judging criteria & $\begin{array}{c}\text { Patients with } \\
\text { meningeal metastasis }\end{array}$ & $\begin{array}{c}\text { Patients with non-tumor } \\
\text { diseases in the brain }\end{array}$ & $Z$ & P-value \\
\hline Criterion 1 & $277.81 \pm 523.21$ & $0.88 \pm 1.25$ & -2.612 & 0.009 \\
Criterion 2 & $243.25 \pm 489.67$ & 0.00 & -3.142 & 0.002 \\
$Z$ & -0.267 & -1.852 & - & - \\
P & 0.531 & 0.062 & - & - \\
\hline
\end{tabular}

with a median age of 62 years (range, 46-76 years). They all pertained to lung adenocarcinoma, and 5 cases were complicated by brain parenchyma metastasis. *Diagnostic criteria for meningeal metastasis: i) Specific tumor history; ii) presence of newly-onset nervous system symptoms and signs; iii) typical enhanced MRI manifestations in the brain; and iv) identification of tumor cells in CSF by cytological examination. The diagnosis could be made if the patient had the former two and the third or the fourth items (6).

The inclusion criteria for the patients with non-tumor diseases in the brain was as follows: i) Patients with non-tumor diseases in the brain, who were admitted to hospital at the same time as those in the experimental group; ii) Patients who required treatment with extra ventricular drainage, a ventriculoperitoneal shunt, lumbar cistern drainage and lumber puncture for CSF examination during hospitalization; iii) providing informed consent. The study was approved by the Ethics Committee of Tianjin Huanhu Hospital (Tianjin, China). According to the above inclusion criteria, a total of 8 patients were enrolled, in which there were 6 males and 2 females with the median age of 40 (35 60 years old). They were all admitted into hospital due to communicating hydrocephalus.

Immuno-FISH. CSF $(7.5 \mathrm{ml})$ was collected from the patients with meningeal metastasis via lumber puncture and stored in the specialized tube for immuno-FISH detection at room temperature. CSF $(7.5 \mathrm{ml})$ was also collected from the patients with non-tumor diseases in the brain through an external ventricular drainage tube and preserved in a specialized tube for immuno-FISH detection at room temperature. The tumor cells were detected within $72 \mathrm{~h}$ through immuno-FISH technology. The detection steps were as follows: i) A negative screening method with immunomagnetic beads was used to enrich the cells: Immunomagnetic beads coated with anti-cluster of differentiation (CD)45 antibody (part of the Human Circulating Rare Cell Subtraction Enrichment kit; Cytelligen, Inc., San Diego, CA, USA) were adopted to remove CD45-positive white cells, and $7.5 \mathrm{ml}$ CSF was added to $100 \mu \mathrm{l}$ cell suspension. ii) Using cell fixative (20\% acetic acid in methanol mixed solution) $100 \mu \mathrm{l}$ of cell suspension was fixed on the slide. Subsequently, centromeric probe 8 (CEP8) was used to detect certain factors for tumor marker-iFISH, including the numbers of chromosome 8, and the expression of PAN-cytokeratin (CK) (which is present in cells derived from the epithelium) and CD45 (which demonstrates that cells are non-leukocytes. The cells were stained with DAPI as they were karyocytes. Cell count was performed using an Olympus-BX53 fluorescence microscope (Olympus
Corporation, Tokyo, Japan) and repeated 5 times. The mean was used as the final value.

Judging criteria for CTCs. DAPI staining was observed under the blue channel using a fluorescence microscope, and blue fluorescence represented karyocytes. CEP8 FISH signals were observed under the orange channel, and the number of orange light spots reflected chromosome 8 number. The majority of chromosome 8 copies in the CTCs were polyploidy, identified by positive CEP8. Chromosome 8 in hematogenous white cells was diploid, identified by negative CEP8. Expression of PAN-CK and CD45 were observed under the green and red channels, respectively, and both green and red fluorescences were identified as being positive. The tumor cells from non-hematogenous epithelial cells were also detected under various channels. CTCs were evaluated according to whether chromosome 8 originated from diploid nucleate, non-hematogenous cells, namely $\mathrm{DAPI}^{+}, \mathrm{CD}^{-} 5^{-}$and $\mathrm{PAN}-\mathrm{CK}^{+}$ or PAN-CK ${ }^{-}$and CEP8.

The judging criteria for CTCs included the following: i) $\mathrm{DAPI}^{+}, \mathrm{CD}^{-} 5^{-}$and $\mathrm{PAN}-\mathrm{CK}^{+}$or $\mathrm{PAN}-\mathrm{CK}^{-}$and $\mathrm{CEP}^{+}$or CEP8; and ii) $\mathrm{DAPI}^{+}, \mathrm{CD}^{-} 5^{-}$and $\mathrm{PAN}-\mathrm{CK}^{+}$or $\mathrm{PAN}_{-} \mathrm{CK}^{-}$and $\mathrm{CEP}^{+}$. The cells pertained to white cells when $\mathrm{DAPI}^{+}, \mathrm{CD}^{4} 5^{+}$, PAN-CK ${ }^{-}$and $\mathrm{CEP}^{-}$were all present (Fig. 1).

Statistical analysis. SPSS v17.0 (SPSS, Inc., Chicago, IL, USA) was used for data analysis. Measurement data were compared using the rank-sum test and were expressed as the mean \pm standard deviation $(x \pm \mathrm{s})$. According to receiver operating characteristic (ROC) curves, the diagnostic sensitivity, specificity, effectiveness, and positive and negative predictive values were calculated. $\mathrm{P}<0.05$ was considered to indicate a statistically significant difference.

\section{Results}

Detection results of CTCs based on different judging criteria. According to criterion 1, CTCs were identified in 12/16 CSF samples from patients with meningeal metastasis and in $2 / 8 \mathrm{CSF}$ samples from the patients with non-tumor diseases in the brain. According to criterion 2, CTCs were detected in 12/16 CSF samples from the patients with meningeal metastasis, while no CTCs were identified in the $8 \mathrm{CSF}$ samples from patients with non-tumor diseases in the brain. Based on the aforementioned criteria, the number of tumor cells in the CSF of patients with meningeal metastasis was significantly higher than those with non-tumor diseases in 
Table II. AUC, standard deviation, P-value and 95\% CI of circulating tumor cells in the diagnosis of meningeal metastasis by immunofluorescence in situ hybridization.

\begin{tabular}{lcccc}
\hline $\begin{array}{l}\text { Judging } \\
\text { criteria }\end{array}$ & AUC & $\begin{array}{c}\text { Standard } \\
\text { deviation }\end{array}$ & P-value & $95 \%$ CI \\
\hline Criterion 1 & 0.824 & 0.085 & 0.011 & $0.657-0.991$ \\
Criterion 2 & 0.875 & 0.072 & 0.003 & $0.705-1.000$ \\
\hline
\end{tabular}

AUC, area under the curve; CI, confidence interval.

Table III. Lower co-ordinate points of ROC curves corresponding to CTCs based on two judging criteria.

\begin{tabular}{lrccc}
\hline Variables & $\begin{array}{c}\text { Diagnostic } \\
\text { points }\end{array}$ & Sensitivity & 1-specificity & $\begin{array}{c}\text { Youden } \\
\text { index }\end{array}$ \\
\hline CTCs based & 1 & 0.750 & 0.625 & 0.375 \\
on criterion 1 & 2 & 0.750 & 0.125 & 0.625 \\
& 3 & 0.688 & 0.000 & 0.688 \\
& 32 & 0.500 & 0.000 & 0.500 \\
& 197 & 0.250 & 0.000 & 0.250 \\
CTCs based & 1,824 & 0.000 & 0.000 & 0.000 \\
on criterion 2 & 1 & 0.750 & 0.000 & 0.750 \\
& 12 & 0.625 & 0.000 & 0.625 \\
& 150 & 0.500 & 0.000 & 0.500 \\
& 1,639 & 0.250 & 0.000 & 0.250 \\
& 12 & 0.000 & $0.000^{\mathrm{a}}$ \\
\hline
\end{tabular}

${ }^{\mathrm{a}}$ Youden index, sensitivity + specificity-1.CTCs, circulating tumor cells.

the brain $(\mathrm{P}=0.009 ; \mathrm{P}=0.002)$ (Table $\mathrm{I})$. The number of tumor cells in the patients with meningeal metastasis and non-tumor diseases in the brain based on criterion 1 was more than that on criterion 2 , but was not statistically significant $(\mathrm{P}=0.531$; $\mathrm{P}=0.062$ ) (Table I).

Diagnostic critical values of CTCs based on different judging criteria. At present, there are no unified evaluation standards or diagnostic critical values regarding the detection of tumor cells in CSF for the diagnosis of meningeal metastasis from lung cancer. According to the aforementioned criteria, the AUCs and critical points of tumor cell detection indexes in the CSF samples were confirmed based on the ROC curves, and the results indicated that the AUC based on criterion 1 was less than that of criterion 2 (Table II; Fig. 2).

The critical point of the maximum correct diagnostic index (Youden index) was regarded as the positive judgement value in the ROC curves. When criterion 1 was used for judging CTCs in the diagnosis of meningeal metastasis, the maximum Youden index was 0.688 , and the diagnostic critical value of CTCs, sensitivity and specificity were 3 tumor cells $/ 7.5 \mathrm{ml}$ CSF, 68.8 and $100.0 \%$, respectively. When criterion 2 was adopted to judge CTCs in the diagnosis of meningeal metastasis, the maximum Youden index was 0.750, and diagnostic

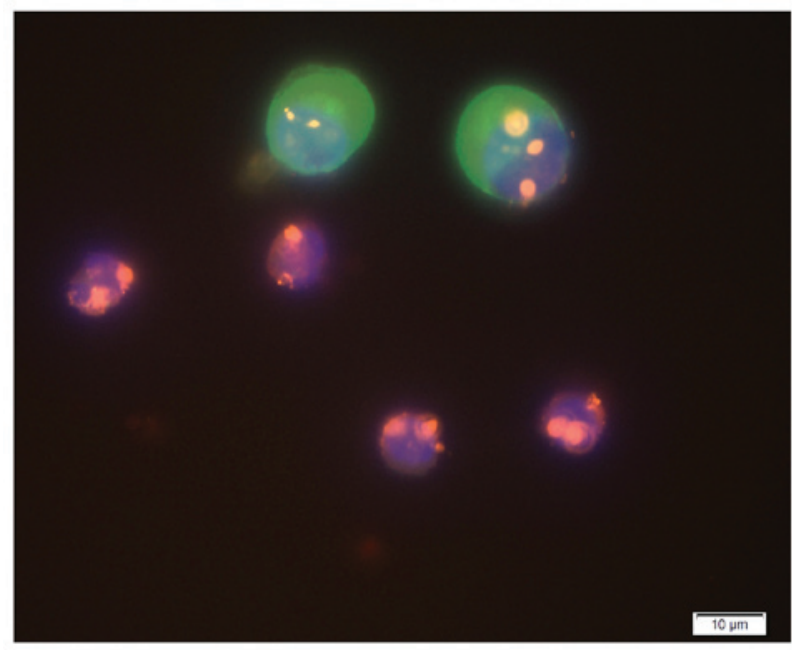

Figure 1. Image of enriched cells under a fluorescence microscope (magnification, x400). Circulating tumor cells: Positive DAPI staining, blue fluorescence; highly expressed PAN-CK, green fluorescence; CEP8, polyploidy; and CD45, no expression. White cells: Positive DAPI staining, blue fluorescence; PAN-CK, no expression; CEP8, diploid; and CD45, red fluorescence. CK, cytokeratin; CD, cluster of differentiation.

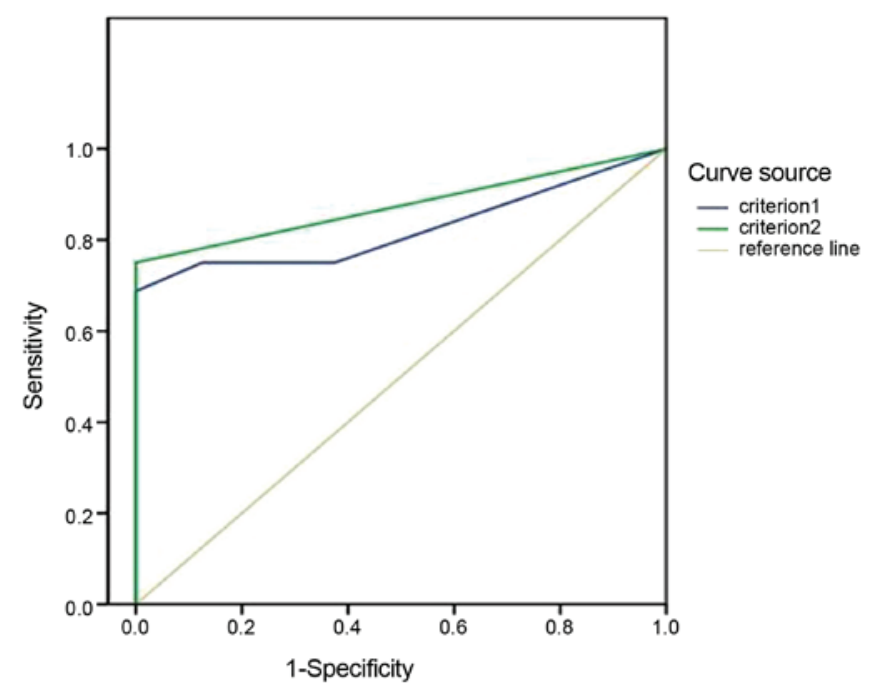

Figure 2. Receiver operating characteristic curves of circulating tumor cells in the diagnosis of meningeal metastasis by immunofluorescence in situ hybridization technology.

critical value of CTCs, sensitivity and specificity were 1 tumor cell/7.5 ml CSF, 75.0 and 100.0\%, respectively (Table III).

Sensitivity, specificity, effectiveness, and positive and negative predictive values of CTCs in CSF in the diagnosis of meningeal metastasis. The specificity, effectiveness, and positive and negative predictive values of CTCs in CSF in the diagnosis of meningeal metastasis based on criterion 2 were all higher than criterion 1, while sensitivity was the same (Table IV).

\section{Discussion}

Meningeal metastasis from lung cancer is characterized by rapid progression of the pathological condition and poor prognosis (2). Early diagnosis and treatment may effectively 
Table IV. Relevant indexes of circulating tumor cells in cerebrospinal fluid in the diagnosis of meningeal metastasis (\%).

\begin{tabular}{lccccc}
\hline $\begin{array}{l}\text { Judging } \\
\text { criteria }\end{array}$ & Sensitivity & Specificity & Effectiveness & $\begin{array}{c}\text { Positive } \\
\text { predictive value }\end{array}$ & $\begin{array}{c}\text { Negative } \\
\text { predictive value }\end{array}$ \\
\hline Criterion 1 & 75.0 & 75.0 & 75.0 & 85.7 & 60.0 \\
Criterion 2 & 75.0 & 100.0 & 83.3 & 100.0 & 66.7 \\
\hline
\end{tabular}

alleviate neurological impairment due to progression (2). At present, the following criteria are primarily adopted to diagnose meningeal metastasis: i) Definite history of tumors; ii) presence of newly-onset nervous system symptoms and signs; iii) typical manifestations of enhanced MRI; and iv) presence of tumor cells via CSF cytology (7). Diagnosis may be made immediately when the former two and the third or fourth items are present. Nevertheless, typical symptoms and signs associated with the central nervous system do not manifest in $>90 \%$ of patients with meningeal metastatic carcinoma (6). Due to the different invasive sites of tumor cells, the clinical manifestations of meningeal metastatic carcinoma are complicated, varied and short of specificity, which makes identification difficult when symptoms caused by brain parenchyma and spinal cord metastases are present, in addition to adverse reactions to treatment for primary tumors. The positive rate of CSF cytology examination was only 55\% through CSF cytology, and a second examination found a rate of $80 \%$; however, performing a third examination did not increased the positive rate any further (8). The specificity of enhanced brain MRI is close to $100 \%$, however, a $65 \%$ false-negative rate and $10 \%$ false-positive rate still exists (9). Therefore, the aforementioned diagnostic methods cannot meet the demand of clinicians in the diagnosis and efficacy evaluation of meningeal metastatic carcinoma. It is thus necessary to identify a detection method with higher sensitivity and specificity. Immuno-FISH detection is able to effectively identify various non-hematogenous epithelial tumor cells in biological fluids by enrichment and analysis techniques. Previous studies have demonstrated that detection of CTCs in CSF samples of patients with meningeal metastasis from lung cancer had higher sensitivity compared to cytology examination $(10,11)$. Therefore, in the present study, immuno-FISH technology was used to detect CTCs in patients with meningeal metastasis and non-tumor diseases in the brain, and its value was investigated in the adjuvant diagnosis of meningeal metastasis from lung cancer.

Clinically, an ideal detection method should possess $100 \%$ specificity and sensitivity, and reliable predictive value; however, it is challenging to apply these methods in clinical settings due to the complicated processes of tumorigenesis, progression and prognosis. Selection of the indexes with higher sensitivity and specificity must respectively lead to increase of false-positive and -negative rates. Positive predictive value refers to the proportion of really-positive patients in the total number of positive cases detected by screening tests. Positive predictive value may reflect the possibility that patients may develop the disease, based on screening results. Negative predictive value refers to the proportion of really-negative population in the total number of negative cases detected by screening tests. The predictive value of diagnostic trials is impacted by sensitivity, specificity and morbidity among the subjects. ROC curves are able to control the false-positive and -negative rates under a small range if it is used to confirm the critical value of diagnostic trials. In the present study, the critical point of the maximum correct diagnostic index (Youden index) was regarded as the judging criterion for positive tumor cells in CSF, and sensitivity and specificity were taken into account to the greatest extent (12). AUC has been widely recognized as a fixed accuracy index that is able to correctly evaluate the diagnostic trials. The AUCs of completely valueless and ideal diagnostic trials are 0.5 and 1 , respectively (13). Nevertheless, it is generally considered that the diagnostic value is lower, moderate and higher if the AUC of this diagnostic trial is 0.5-0.7, 0.7-0.9 and >0.9, respectively (13).

In the current study, the difference between criterion 1 and 2 was whether non-hematogenous cells with negative chromosome 8 copies were determined as CTCs. The number of tumor cells in the patients with meningeal metastasis and non-tumor diseases in the brain based on criterion 1 was more than that of criterion 2 , but was not statistically significant $(\mathrm{P}>0.05)$. In the diagnosis of meningeal metastasis, each index of tumor cells in CSF based on criterion 2 was superior to criterion 1 . The sensitivities of the CTCs were $75 \%$ in the diagnosis of meningeal metastasis according to two detection criteria, but based on criterion 2, the diagnostic specificity, effectiveness, and positive and negative predictive values of the CTCs in CSF were higher in the diagnosis of meningeal metastasis from lung cancer. Therefore, it is recommended to use criterion $2\left(\mathrm{DAPI}^{+}, \mathrm{CD}^{-} 5^{-}\right.$and $\mathrm{PAN}-\mathrm{CK}^{+}$or PAN-CK ${ }^{-}$and $\mathrm{CEP}^{+}$) to evaluate CTCs in CSF. Additionally, non-hematogenous cells with negative chromosome 8 may be deciduous meningocytes or epidermal cells, arising as a result particular clinical operations, including lumber puncture and extra ventricular drainage, that enter into the CSF samples and consequently lead to an increase in the diagnostic false-positive rate. However, whether removal of these types of cells is able to increase the false-negative rate due to a small sample size, as in the present study, remains to be elucidated.

In conclusion, when criterion 2 was adopted by the current study to judge CTCs in the diagnosis of meningeal metastasis, the AUC, 95\% CI, P-value and maximum of Youden index were $0.875,0.705-1.000,0.003$ and 0.750 , respectively. The diagnostic critical value of CTCs was 1 tumor cell/7.5 ml CSF. Namely, when one CTC was identified in $7.5 \mathrm{ml} \mathrm{CSF}$ from patients with meningeal metastasis, the sensitivity, specificity, effectiveness, and positive and negative predictive values of diagnosing meningeal metastasis were 75.0, 100.0, 83.3, 100.0 and $66.7 \%$, respectively. Therefore, detection of tumor cells in CSF has better clinical value in the diagnosis of meningeal 
metastasis from lung cancer via immuno-FISH technology. As a small sample size was used in the current study, further clinical experiments are required to verify whether multiple detections of one sample is able to increase the diagnostic sensitivity. Further study is of great importance for the detection of CTCs in CSF in the evaluation of efficacy and prognosis.

\section{Acknowledgements}

The present study received funding from the Tianjin Municipal Health Bureau, which supports science and technology projects (no. 2014kz042).

\section{References}

1. Leal T, Chang JE, Mehta M and Robins HI: Leptomeningeal metastasis: Challenges in diagnosis and treatment. Curr Cancer Ther Rev 7: 319-327, 2011.

2. Nagpal S, Riess J and Wakelee H: Treatment of leptomeningeal spread of NSCLC: A continuing challenge. Curr Treat Options Oncol 13: 491-504, 2012.

3. Yu Y, Chen Z, Dong J, Wei P, Hu R, Zhou C, Sun N, Luo M, Yang W, Yao R, et al: Folate receptor-positive circulating tumor cells as a novel diagnostic biomarker in non-small cell lung cancer. Transl Oncol 6: 697-702, 2013.

4. Igawa S, Gohda K, Fukui T, Ryuge S, Otani S, Masago A, Sato J, Murakami K, Maki S, Katono K, et al: Circulating tumor cells as a prognostic factor in patients with small cell lung cancer. Oncol Lett 7: 1469-1473, 2014
5. Romiti A, Raffa S, Di Rocco R, Roberto M, Milano A, Zullo A, Leone L, Ranieri D, Mazzetta F, Medda E, et al: Circulating tumor cells count predicts survival in colorectal cancer patients. J Gastrointestin Liver Dis 23: 279-284, 2014.

6. Ma C, Jiang R, Li J, Wang B, Sun L and Lv Y: Research progress of lung cancer with leptomeningeal metastasis. Zhongguo Fei Ai Za Zhi 17: 695-700, 2014 (In Chinese).

7. Wang Y, Gao Y, Zhu YF and Tao RJ: The diagnosis and treatments progress of meningeal carcinomatosis. Zhongguo Lin Chuang Shen Jing Wai Ke Za Zhi 18: 760-762, 2013.

8. Le Rhun E, Massin F, Tu Q, Bonneterre J, Bittencourt Mde C and Faure GC: Development of a new method for identification and quantification in cerebrospinal fluid of malignant cells from breast carcinoma leptomeningeal metastasis. BMC Clin Pathol 12: 21, 2012.

9. Clarke JL, Perez HR, Jacks LM, Panageas KS and Deangelis LM: Leptomeningeal metastases in the MRI era. Neurology 74: 1449-1454, 2010

10. Jiang R, Ma CH, Zhu ZL, Li JD, Wang B, Sun LW and Lv Y: Application of circulating tumor cell detection in cerebrospinal fluid in the diagnosis of meningeal metastasis from non-small cell lung cancer. Chin J Contemp Neurol Neurosurg 14: 698-701, 2014.

11. Ma CH, Jiang R, Li JD, Wang B, Sun LW and Lv Y: A new method for enrichment and calculation of malignant cells in cerebrospinal fluid of patients with meningeal metastasis from lung cancer. Tianjin Medical Journal 43: 419-421, 2015.

12. Wang P, She CH, Li P, Pu YZ, Wang XG and Li WL: Application of tumor markers in the adjuvant diagnosis of meningeal metastasis from lung cancer. Chin J Clin Oncol 35: 61-64, 2008.

13. Wang JH: Application of ROC curve in clinical diagnostic experiments. Chin J Hypertens 16: 175-177, 2008. 